\title{
Virtual Instrument Design of Metro Power Harmonic Detection System Based on the Improved FFT Algorithm
}

\author{
Y.B. Ma \\ Xi'an Metro Co., Ltd. \\ China
}

\begin{abstract}
The current hardware detection methods have many drawbacks, such as poor flexibility and single function. Considering the frequency fluctuation and halfwave symmetric characteristic of the metro power system signals, an improved FFT algorithm that only analyzes the odd harmonics in its calculation is presented and just has a half amount of the traditional FFT. On this basis, the metro power harmonic detection system was developed based on virtual instrument. The PC-DAQ structure scheme was adopted for hardware of this system.The software of this system was developed based on LabVIEW2009. The tests show that this system can achieve the expecting functions and has the high accuracy.
\end{abstract}

Keywords-the improved FFT algorithm; harmonic analysis; virtual instrument

\section{INTRODUCTION}

Increasing power capability and nonlinear loads, the harmonic pollute become more and more serious, directly affect the efficiency and safety of the metro power, and reduce the quality of power grid. The DC1500V/750V is often provided for traction power system in metro,which adopt $12 / 24$ pulse rectifier whose high harmonics mainly are 5 times, 7 times, 11 times, 13 times, 23 times, 25 times harmonics. It is clear that half-wave symmetric characteristic exist in metro power system. So it is very important to improve the metro power quality by monitoring the odd harmonics fast and accurately.This paper present a improved FFT to detect harmonics signals and design the harmonics monitor system that is realized by the virtual instrument technology. There are some functions in this system,such as rate calculation of each harmonics voltage, total harmonics distortion calculation.

\section{THE IMPROVED FFT}

The FFT algorithm is continuous unfolded by even and odd frequency in every level until two point DFT, $\mathrm{N}$ point FFT need $N / 2 * \log _{2} N$ complex multiplication, and $N * \log _{2} N$ complex add. The FFT calculation formulation from $\mathrm{N}$ to $\mathrm{N} / 2$ is

\author{
Z.G. Ye \\ MXTronics Corporation \\ China
}

$$
\begin{aligned}
& X(2 r)=\sum_{n=0}^{N / 2-1} x_{1}(n) W_{N / 2}^{n r} \\
& X(2 r+1)=\sum_{n=0}^{N / 2-1} x_{2}(n) W_{N / 2}^{n r} \\
& x_{1}(n)=x(n)+x(n+N / 2) \\
& x_{2}(n)=[x(n)-x(n+N / 2)] W_{N}^{n}
\end{aligned}
$$

In which, $0 \leq n \leq N / 2-1,0 \leq r \leq N / 2-1$.

Considering half-wave symmetric characteristic, this paper improved the traditional FFT, only resolved the odd sequence $X(2 r+1)$, omit the even sequence $X(2 r), X(2 r+1)$ is resolved again until two point, the resolve formulation is

$$
\begin{aligned}
& X(4 l)=\sum_{n=0}^{N / 4-1} x_{3}(n) W_{N / 4}^{n r} \\
& X(4 l+1)=\sum_{n=0}^{N / 4-1} x_{4}(n) W_{N / 4}^{n r} \\
& x_{3}(n)=x(n)+x(n+N / 4) \\
& x_{4}(n)=[x(n)-x(n+N / 4)] W_{N / 2}^{n}
\end{aligned}
$$

In which, $0 \leq n \leq N / 4-1,0 \leq r \leq N / 2-1$.

It is clear that the improved FFT [1]first resolved only odd frequency $X(2 r+1)$, and then resolved even and odd frequency every level until only two point DFT. The calculate amount decrease by half. $\mathrm{N}$ point sample DFT only need $N / 4 * \log _{2} N$ complex multiplication, and $N / 2 * \log _{2} N$ complex add, which greatly enhance the real-time feature.

\section{SYSTEM DESIGN AND IMPLEMENTATION}

This paper introduce the virtual instrument technology, use the LabVIEW soft to design a metro power grid harmonics monitor system based on the improved FFT.

\section{A. Hardware Design}

The hardware includes voltage and current sensors, signal 
conditioning, data acquire cards, and PC as shown in Fig 1.

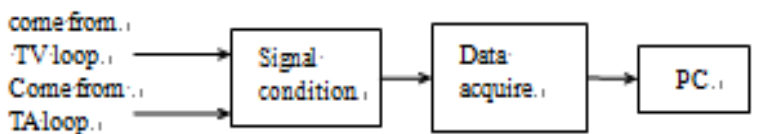

FIGURE I. STRUCTURE OF HARDWARE SYSTEM BASED ON VIRTUAL INSTRUMENT.

The current and voltage signal generated from the secondary of mutual inductance unit. SC-2345 signal condition box of NI is used to isolation, filter, and amplify the signal. At last, the signal is transmitted to PCI-6024E that is a data acquire card -16 channels with 12bits resolution ADC, two 24bit counters, $200 \mathrm{KHz}$ sample rate-easy to meet the $50 \mathrm{~Hz}$ signal band require of power grid.

\section{B. Soft Design}

According to the condition of metro power system, the monitor system must meet the IEC and national power quality A level require, which are GB/T15945-1995 and GB/T145491993. So the soft structure must provide the important functions that is date acquisition,analysis and processing, realtime display. Its program graph is shown in Fig 2.Analysis and processing is the critical part of the whole soft. Take A phase voltage for example, synchronous sample is implemented by self-adapting frequency tracking[2][3] and improved FFT algorithm. Once the frequency have been tracked, harmonics analysis algorithm based on improved FFT calculate the phase and amplitude of each harmonics, the rate of harmonics and the total harmonics distortion.

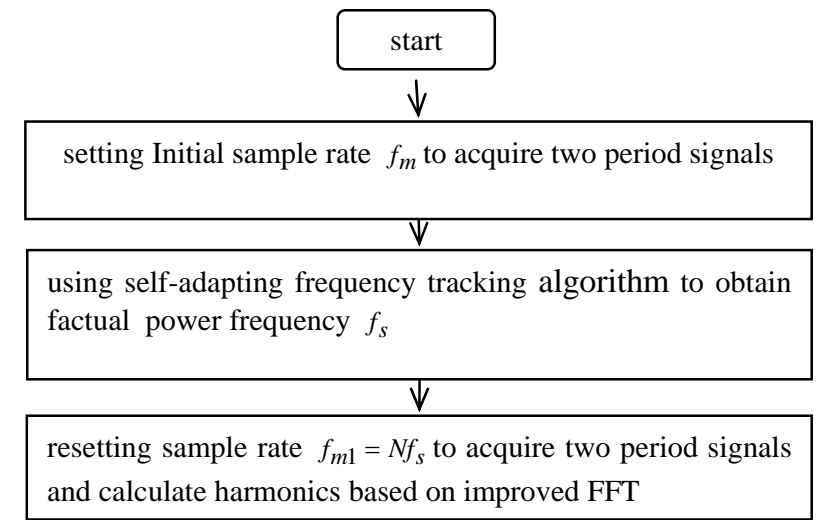

$\downarrow$

displaying the voltage, current, spectrum etc.

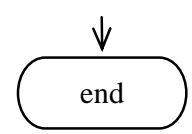

FIGURE II. THE STRUCTURE OF SOFT PROGRAM.

\section{TEST THE SOFT FUNCTION}

Suppose the A phase voltage is

$$
\begin{aligned}
& U=220 * \sin \left(2 \pi f_{1} t\right)+\frac{1}{3} * 220 * \sin \left(2 \pi \pi_{3} t\right)+\frac{1}{5} * 220 * \sin \left(2 \pi \pi_{5} t\right)+ \\
& \frac{1}{10} * 220 * \sin \left(2 \pi f_{7} t\right)+\frac{1}{20} * 220 * \sin \left(2 \pi f_{11} t\right)+\frac{1}{40} * 220 * \sin \left(2 \pi f_{23} t\right)
\end{aligned}
$$

and no harmonics in $\mathrm{B}$ and $\mathrm{C}$ phase voltage, if the frequency of $U$ changed from $50 \mathrm{~Hz}$ to $46 \mathrm{~Hz}$ in $\mathrm{t}=0.02$. In this same condition, the improved FFT, self-adapting frequency tracking and system functions are tested by labVIEW [4][5] as shown in Fig3.
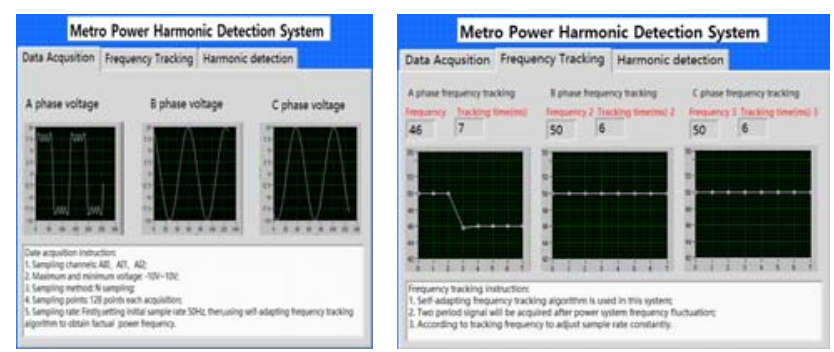

(a) Test interface of data acquire

(b) Test interface of frequency tracking

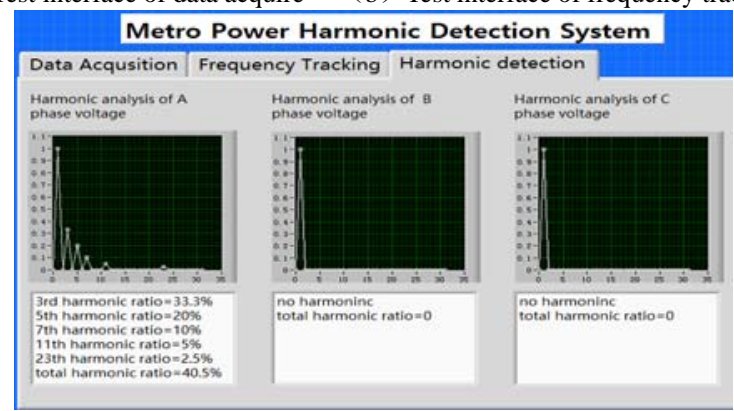

(c) Test interface of harmonics analysis and process

FIGURE III. TEST INTERFACE OF HARMONICS ANALYSIS AND PROCESS SYSTEM OF METRO POWER GRID.

From the test result, each module can work well, the improved FFT can decrease the calculate mount, enhance the real-time performance, and the accuracy of the frequency tracking, amplitude calculate, harmonics analysis all can meet the design require. The tracked frequency is $45.998 \mathrm{~Hz}$, tracking error is $0.1 \%$, tracking time is only $7 \mathrm{~ms}$. So this detect system is high accuracy and perfect in real-time.

\section{CONCLUSION}

Firstly according to half-wave symmetric characteristic exist in metro power system, a improved FFT is presented ,which is half amount of traditional FFT and reduce operation time.At the same time, a new detect system is implemented using virtual instrument technology based on the improved FFT. It can acquire data real-time, track the base wave frequency, harmonics analysis, and display the graph and table, which well resolve the problem of high cost, single function, bad flexibility compared with the traditional hardware detect system. The system tests show that this system can achieve the expecting functions and has the advantages of high accuracy.

\section{REFERENCES}

[1] Liu Yan.A class of hafl-wave symmetry chracteristice of the grid harmonic detection method research[J].uournal of shannxi university of sicence \& technology,2012,10:104-107. 
[2] Zhang Bin,Zhang Dong-lai. Adaptive fudamental component extraction and grequency tracking algorithm for power systems[J].Proceedings of the CSEE,2011,31(25):82-86.

[3] Mai Rui-kun,He Zheng You.Adaptive frequency tracking algorithm for power systems[J],Proceedings of the CSEE ,2010,30(16), 73-78.

[4] Qi Chao,Liu Wei-jian,Wang Yan.Power quality monitoring system based on LabVIEW[J].Jouranl of harbin uniersity of commerce,2014,30(4):469-472.

[5] Zhao Si-jun ,Shan Lei. Harmonic monitoring system of coal mine grid based on virtual instrument[J].Industry and mine automation,2010,9:6870. 